\title{
Thermonuclear Supernovae
}

\section{F. K. Röpke*}

Max-Planck-Institut für Astrophysik, Karl-Schwarzschild-Str. 1, D-85741 Garching, Germany

E-mail: fritz@mpa-garching.mpg.de

The application of Type Ia supernovae (SNe Ia) as distance indicators in cosmology calls for a sound understanding of these objects. Recent years have seen a brisk development of astrophysical models which explain SNe Ia as thermonuclear explosions of white dwarf stars. While the evolution of the progenitor is still uncertain, the explosion mechanism certainly involves the propagation of a thermonuclear flame through the white dwarf star. Three-dimensional hydrodynamical simulations allowed to study a wide variety of possibilities involving subsonic flame propagation (deflagrations), flames accelerated by turbulence, and supersonic detonations. These possibilities lead to a variety of scenarios. I review the currently discussed approaches and present some recent results from simulations of the turbulent deflagration model and the delayed detonation model.

Supernovae: lights in the darkness (XXIII Trobades Científiques de la Mediterrània) October 3-5 2007

Mao, Menorca, Spain

\footnotetext{
* Speaker.
} 


\section{Introduction}

Thermonuclear supernova explosions are an astrophysical model for the astronomical class of Type Ia supernovae (SNe Ia henceforth). These objects are of interest in many fields of astrophysics and astronomy. Being one of the main sources of iron group elements, SNe Ia contribute to the chemical evolution of galaxies (e.g., [1]). They affect star formation and drive shock waves in the interstellar and intergalactic media.

The main driver of SN Ia research over the past years, however, has been their application in observational cosmology. Here, SNe Ia were employed as distance indicators (as put forward by [2]). At redshifts above 0.5 , a significant deviation from the linear Hubble law was noticed which led to the spectacular interpretation of the Universe currently undergoing an accelerated expansion [3,4]. The determination of the force driving this acceleration is perhaps one of the greatest challenges in contemporary physics. For the time being, it is parametrized as "dark energy" (e.g., [5]). The simplest form of dark energy is a cosmological constant, but more complicated contributions to the energy-momentum tensor in the Einstein equations are also conceivable. A first step to determine the nature of dark energy would be to constrain its equation of state. SNe Ia seem to be a suitable tool for this task and currently two major campaigns $[6,7]$ apply them in distance determinations of hundreds of supernovae out to redshifts of $z \sim 1$. The large number of observations is necessary to reduce the statistical errors because putting tight constraints on dark energy equation of state requires a high accuracy of the distance determinations.

From a theoretical point of view, however, the applicability of SNe Ia as distance indicators is still not satisfactorily answered. SNe Ia are remarkably uniform events by astrophysical standards, but evidently no standard candles. Only a calibration of the distance measurements according to empirical correlations between observables provides the necessary accuracy for the determination of cosmological parameters. Such calibrations may be afflicted with systematic errors. Being derived from a set of nearby well-observed SNe Ia, there is no guarantee that they perform well for supernovae at high redshifts, too. Getting a handle on these uncertainties is one of the goals of modeling SNe Ia.

\section{Astrophysical modeling}

The cornerstones of the astrophysical model of SNe Ia derive from the fundamental characteristics of these events:

- Evidently, SNe Ia belong to the most energetic cosmic explosions, releasing about $10^{51} \mathrm{erg}$ of energy. For a short period of time they can outshine an entire galaxy consisting of tens of billions of stars.

- SNe Ia spectra are characterized by lacking indications of hydrogen and helium which together with a pronounced P Cygni silicon line at maximum light classifies these objects [8]. Lines of intermediate-mass elements (IME, such as $\mathrm{Si}, \mathrm{Ca}, \mathrm{Mg}$ and $\mathrm{S}$ ) and oxygen are observed in near-maximum light spectra (e.g., [9]).

- SNe Ia form a class of remarkable homogeneity with respect to observed lightcurves and spectra (e.g., [2]). 
Assuming supernovae to generally originate from single stellar objects, only their gravitational binding energy, released in a collapse towards a compact object [10], or its nuclear energy, released in explosive reactions [11], come into consideration as possible energy sources. In the particular case of SNe Ia, no compact object is found in the remnants excluding the first possibility. The homogeneity of the class of SNe Ia and the fact that no hydrogen is found in their spectra provides a strong hint that the object undergoing the nuclear explosion may be a white dwarf (WD) star consisting of carbon and oxygen $(\mathrm{C}+\mathrm{O})$.

Lightcurves of SNe Ia rise over a time scale of several days and decline over months. It is therefore clear that they cannot be powered directly by the explosion since the temperatures fall off much too rapidly in the expansion. This problem was solved by noting that the ${ }^{56} \mathrm{Ni}$ produced in large amounts in the explosive thermonuclear burning provides the energy source for the optical event by radioactive decay to ${ }^{56} \mathrm{Co}$ and ${ }^{56} \mathrm{Fe}[12,13]$.

\subsection{Progenitor evolution and ignition}

A single WD is an inert object. How can it reach an explosive state? The only way to introduce the necessary dynamics into the system is to assume it to be part of a binary system and to gain matter from the companion. Several models have been proposed for this progenitor evolution. Here, we will discuss only models resulting from the so-called single-degenerate Chandrasekharmass scenario, which has received by far most attention recently. For a summary of alternative models, we refer to [14].

In the single degenerate scenario $[15,16,17]$, the WD accretes matter from a non-degenerate companion (either a main sequence star, or an AGB star). This idea was recently supported by the detection of the potential companion of Tycho Brahe's supernova of 1572, which is a solar-type star [18]. By accreting material from this companion and burning it hydrostatically at the surface, the WD may reach the Chandrasekhar mass $M_{\mathrm{Ch}}$. Limiting the fuel available in the explosion to $M_{\mathrm{Ch}} \sim 1.4 \mathrm{M}_{\odot}$, this Chandrasekhar-mass model appears particularly favorable since it provides a natural explanation for the striking uniformity of SNe Ia in the gross observational features. At the same time, it is afflicted with great uncertainties. Achieving a stable mass transfer in the progenitor binary system to build up a Chandrasekhar mass WD is highly non-trivial (e.g., [19]) and the observational evidence for such systems is sparse.

When the WD approaches the Chandrasekhar limit, the density at the center of the WD increases rapidly so that fusion of carbon ignites. Contrary to the situation in main sequence stars, the degenerate material of the WD does not allow for moderation of the burning by expansion. Heat transport is achieved here by convection giving rise to a stage of convective carbon burning that lasts for several hundred years. This phase is terminated by one or more small spatial regions undergoing a thermonuclear runaway, marking the birth of a thermonuclear flame and the onset of the explosion. The convective burning stage and the conditions at flame ignition are extremely hard to model both analytically and numerically. Therefore the exact shape and location of the first flame spark(s) is not yet well constrained [20, 21, 22, 23, 24]. Does ignition occur in a single spot or in multiple sparks with a stochastic distribution? Does it take place at the center of the WD or do pre-ignition convective motions lead to large asymmetries? Evidently, the ignition structure is a crucial initial parameter in multi-dimensional explosion models [25, 26, 27, 28, 29, 30]. 


\subsection{Flame propagation and explosion}

The goal of SN Ia explosion models is to follow the propagation of the thermonuclear flame from its ignition near the center of the WD outwards and to determine the nuclear energy release and the structure of the ejected material.

Hydrodynamics allows for two distinct modes of flame propagation. One is the subsonic deflagration in which the flame is mediated by the thermal conduction of the degenerate electron gas and the other is a supersonic detonation in which the burning front is driven by shock waves. Either one of these modes or a combination of both have been suggested in different explosion models:

- The prompt detonation model [31] attempts to explain SNe Ia by a detonation ignited at the center of the WD and propagating outward. This produces enough energy for a SN Ia event. However, ahead of a supersonic detonation wave, the fuel cannot expand and is therefore incinerated at the high densities of an equilibrium white dwarf. This results in the almost complete conversion of the material to nickel-peaked nuclear statistical equilibrium [31], which is in conflict with the intermediate mass elements observed in SN Ia spectra. Such nucleosynthetic problems rule out pure detonations as a standard model for SN Ia explosions.

- The deflagration model [32] assumes the flame to propagate in the subsonic deflagration mode. The laminar burning speed of the deflagration flame is determined by microphysical transport processes. For conditions of carbon burning in $\mathrm{C}+\mathrm{O}$ WDs it is highly subsonic [33] and therefore the flame propagates far too slowly to explain SN Ia explosions. The expansion of the star quenches burning before the WD gets unbound. On the other hand, this model can cure the problem of nucleosynthesis, since rarefaction waves travel ahead of the flame with sound speed and lower the fuel density prior to burning. Thus, the material can partly be processed into intermediate mass elements. The deflagration model undergoes a significant improvement when multidimensional effects are taken into account as will be discussed in detail in Sect. 4.

- The delayed detonation model $[34,35,36]$ unites the advantages of the deflagration and the detonation models. Burning starts out in the slow deflagration mode pre-expanding the star. At some point, a transition from the initial subsonic deflagration to a supersonic detonation takes place. This detonation is an easy way to explain the energy release necessary for a SN Ia explosion. The important notion in this model is that a detonation in low density fuel (pre-expanded in the deflagration stage) can lead to only partial burning and is therefore capable of generating intermediate mass elements. A detailed account of this model will be given in Sect. 5.

- The pulsational delayed detonation model [37] is similar to the delayed detonation model in the sense that it combines an initial deflagration with a later detonation. The flame is assumed to propagate in the initial deflagration phase with its laminar burning speed and pre-expands the star. Due to the slow flame velocity, the burning front stalls and fails to unbind the star. The WD then re-contracts giving the interface between burnt and unburnt material enough 
time to mix and to become nearly isothermal. Compressional heating finally triggers a detonation at densities that are lower than that prior to the first expansion phase. The assumption of the flame propagating with the pure laminar burning velocity in the deflagration phase originally made in this model seems unrealistic because of the flame instabilities and the resulting turbulent flame acceleration. Recent multidimensional deflagration models [38, 39] demonstrated that taking these effects into account, the star is likely to get unbound instead of recontracting.

\section{Numerical modeling}

Numerical models of SN Ia explosions have to face three major challenges. The vast range of relevant length scales cannot be resolved in computational models in the foreseeable future. It extends from the radius of the WD star $(\sim 2000 \mathrm{~km}$ at the onset of the explosion and expanding in the process) down to the flame width which is well below one centimeter and, including turbulence effects, to the Kolmogorov scale of less than a millimeter. Apart from this problem, numerical models need to take into account inherently three-dimensional physical phenomena and to solve the equations of nuclear burning. To meet all these requirements in a single simulation will be impossible in the foreseeable future. Therefore the problem has been tackled in different approaches.

The first path towards SN Ia explosion modeling is to restrict the simulations to only one spatial dimension. Here, in principle a resolution of the relevant scales is achievable and a detailed description of the nuclear reactions is feasible. However, this approach fails to consistently incorporate crucial three-dimensional physical mechanisms.

In multi-dimensional simulations (see [40, 41] for recent reviews), the computational costs of modeling the explosion hydrodynamics is prohibitive to directly resolve all relevant scales as well as details of the nuclear processes. While the latter may be improved in the forthcoming years, and is meanwhile separated from the actual explosion simulations still maintaining a reasonable accuracy (for an approach based on reconstructing the nucleosynthesis from tracer particles, see $[42,43])$, even a drastic increase in computational capabilities will not allow for a resolution of all relevant scales in multi-dimensional simulations. Consequently, additional modeling effort is required in order to implement a consistent description of flame propagation in these simulations (see Sect. 4).

A third approach is to study specific effects on a limited range of spatial scales, in order to validate assumptions and improve modeling techniques of the large-scale SN Ia simulations.

\subsection{Objectives of modeling Type Ia supernovae}

Three-dimensional numerical models of Type Ia supernovae strive for a self-consistent astrophysical description of these astronomical events. The goal is to achieve a viable model by starting out from fundamental physical laws ("first principles") and to formulate the model without introducing free parameters. Avoiding tunable parameters allows the simulations to gain a high level of predictive power. Only this way, a direct comparison of synthetic observables derived from the simulations with actual SN Ia observations facilitates a thorough validation of the modeling approach. 
This aim of multidimensional models is significantly different from earlier one-dimensional modeling approaches. Here, the flame propagation velocity, which is determined by inherently multidimensional effects such as instabilities and turbulences, cannot be consistently determined. It thus introduces a free parameter which has significant impact on the result of the simulated explosion. With such a powerful tunable parameter an impressive agreement between one-dimensional simulations and observations could be achieved. This in turn tells us a great deal about the global properties of the explosion mechanism. Successful one-dimensional models (e.g. W7 [44]) bring forward the average flame velocity that needs to be attained in different stages of the explosion and remain the benchmark of the (spherically averaged) chemical composition of the explosion ejecta.

Successful multidimensional models, however, would diverge from this way of tackling SN Ia modeling as an inverse problem. Modeling the explosion from first principles, they make predictions of observables. This paves the way to investigating questions related to the cosmological use of SNe Ia: What is the precision of SNe Ia as distance indicators? What is the reason for the SN Ia diversity? How does the diversity relate to variations in the properties of the progenitor system? How do these variations translate (via the explosion process) to observable features? How can one improve the calibration techniques of SN Ia cosmology? With ongoing observational campaigns, projects currently starting and future surveys, the number of observed $\mathrm{SNe}$ Ia will increase from currently a few hundred per year to several thousands. This calls for a sound theoretical understanding of SNe Ia as an astrophysical phenomenon. A rapid development of the field of multidimensional SN Ia simulations gives hope that these models will be able to tackle the questions raised above in the not too far future.

\subsection{Current status of SN Ia modeling}

The zoo of variants of the Chandrasekhar-mass single-degenerate SN Ia model recently studied in multidimensional simulations comprizes

- the turbulent deflagration model (see also Sect. 4), which is so-far best studied in multidimensional simulations $[38,45,39,46,47,25,26,28,48]$. The advantage of this model is that the explosion process can be formulated free of tunable parameters. Therefore it is possible to compare the outcome of simulations directly to observations in order to assess the validity of the model. Systematic tests of the initial parameters of the model on the outcome of the exploson process $[49,43]$ and their implication for SN Ia cosmology [50] are possible.

- the delayed detonation model [51, 52, 53, 54], which mainly suffers from the uncertain mechanism providing a deflagration-to-detonation transition in thermonuclear supernovae. Fixing this uncertain parameter to a physically motivated hypothesis, however, the results of corresponding numerical simulations look promising (see Sect. 5).

- the gravitationally confined detonation model, which arises from asymmetric deflagration flame ignitions. It has been claimed that in some cases the deflagration may fail to unbind the WD [27]. As a consequence of the off-center ignition, the ashes of the deflagration burning quickly float towards the surface of the WD. If still gravitationally bound, they start to sweep around the unburnt core of the star and collide on the far side. This collision has been claimed to trigger a detonation wave $[27,55,56]$ (see, however, $[28,57]$ ) propagating 
inwards and burning out the core of the WD. Very energetic and bright events are expected from such a model.

- the pulsational reverse detonation model, which follows the pulsational phase of the gravitationally bound WD if no detonation is triggered by the collision of ashes as in the gravitationally confined detonation scenario. Pulsational contraction has been suggested to trigger a detonation reviving the explosion in this case [58].

The goal of a fully self-consistent SN Ia model that agrees with all observatonal features is not reached yet. Some of the modeling approaches, however, seem very promising. The following part of the paper focuses on a detailed description of the first two of the above listed models.

\section{The turbulent deflagration model}

The turbulent deflagration model extends the laminar deflagration model by considering effects of instabilities and turbulence on the flame propagation. These lead to a significant acceleration of the burning and to viable explosions of the WD star.

\subsection{Turbulent deflagratlion in thermonuclear supernovae}

The major effect accelerating the flame is due to the buoyancy unstable flame propagation from the center of the star outwards. It leaves behind light and hot ashes below the dense fuel - a density statification inverse to the gravitational acceleration. In its non-linear stage, the RayleighTaylor instability leads to the formation of mushroom-shaped burning bubbles raising into the fuel. The Reynolds number typical for this situation is as high as $10^{14}$. Clearly, shear (KelvinHelmholtz) instabilities at the interfaces of these bubbles will generate turbulent eddies which then decay to smaller scales forming a turbulent energy cascade. The flame will interact with these eddies down to the Gibson-scale at which the turbulent velocity fluctuations become comparable to the laminar flame speed. Below the Gibson scale, the flame burns faster through turbulent eddies than they can deform it, and the flame propagation is thus unaffected by turbulence there. This interaction corrugates the flame increasing its surface, enhancing the net burning rate, and, consequently, accelerating the effective flame propagation speed.

In the numerical implementation of the deflagration model, the relevance of turbulent effects amplifies the scale problem since the turbulent cascade extends to the extremely small Kolmogorov scale $(<1 \mathrm{~mm})$ where the turbulent energy is dissipated into heat. The flame interaction with the turbulent cascade down to the Gibson scale must be taken into account. Current 3D simulations capturing the entire star reach resolutions around one kilometer while the Gibson scale is of the order of $10^{4} \mathrm{~cm}$ at the beginning of the explosion and decreases steadily. For large-scale multidimensional SN Ia simulations this has three consequences:

1. The internal flame structure cannot be resolved. Thus, an effective flame model has to be applied and complementary small scale simulations are required.

2. It is not possible to fully resolve the interaction of the flame with turbulence. Therefore modeling of the effects on unresolved scales is necessary. 
3. Assumptions about the flame properties on unresolved scales $[59,49,60,61,62]$ have to be validated in separate small-scale simulations.

Multi-dimensional models are inevitable to consistently determine the turbulent flame propagation velocity. Given the wide range of scales on which the flame is affected by turbulence, this is an ambitious project, additionally challenged by the lack of resolution of the thermonuclear flame structure. For both problems, different approaches have been taken, guided by the theory of turbulent combustion in terrestrial flames (see [63]).

\subsection{Flame model}

Two major strategies to tackle the problem of the unresolved internal flame structure can be distinguished. On the one hand, a flame capturing technique [64] mimics flame propagation by an artificial diffusion mechanism which broadens the internal flame structure to a certain number of computational grid cells. It has been applied in SN Ia explosion simulations [39, 51, 65, 27, 56].

On the other hand, a completely different approach [66] treats the flame as a sharp discontinuity separating the fuel from the ashes. It is numerically represented applying the level-set technique [67]. Here, the flame front is associated with the zero level set of a scalar function $G$ representing the distance from the interface. A model for flame propagation based on this technique [68] was modified for thermonuclear flames in SN Ia explosions [66, 69]. This scheme was applied in a number of simulations $[45,38,70,46,47,71,72,26,43,25,28,48]$, and the simulations presented below are based on it.

\subsection{Turbulent combustion model}

The wide range of scales involved in turbulent combustion in thermonuclear supernovae makes direct simulations virtually impossible. Only parts of the flame/turbulence interaction range and of the resulting flame surface enlargement can be resolved on the computational grid. This deficit is usually compensated by attributing an effective turbulent flame speed $s_{\mathrm{t}}$ to the unresolved flame front, which must be determined by theoretical considerations.

One of the cornerstones of the theoretical description of turbulent combustion is the notion of different regimes of flame/turbulence interaction [73]. These regimes are distinguished by the ability of turbulent eddies to penetrate the internal flame structure. Since the Gibson scale is much larger than the flame width for most parts of the SN Ia explosion, this will not be the case here and accordingly the combustion falls into the regime of wrinkled and corrugated flamelets (e.g., [63]). In this regime, the full flame structure is corrugated by the interaction with turbulence and the resulting surface enlargement accelerates its propagation. The flame propagation completely decouples from the microphysics of the burning for sufficiently strong turbulence [74]. It is entirely determined by the turbulent velocity fluctuations, that is, the effective turbulent flame speed $s_{\mathrm{t}}$ is proportional to the turbulent velocity fluctuations $v^{\prime}$.

One of the challenges of deflagration models of SN Ia explosions is thus to determine these velocity fluctuations correctly. Since the resolution in multi-dimensional simulations is insufficient to resolve the phenomena directly, modeling approaches have to be taken. The assumption of the flame propagation being entirely driven by buoyancy instabilities $[39,65,27,56]$ falls short of 
reproducing the effects of a turbulent cascade which will develop due to large-scale shear instabilities and dominate the turbulence properties at small scales. Such effects are taken into accunt only with appropriate subgrid-scale turbulence models [75, 72]. Guided by the technique of Large Eddy Simulations (LES), this model determines the turbulence energy on unresolved scales based on conservation laws. It is applied in the simulations discussed below.

In the very late stages of the SN Ia explosion, the fuel density drops due to the expansion of the WD to values where the flame width becomes broader than the Gibson length. Then, turbulence penetrates the internal structure of the flame and it enters the regime of distributed burning [73]. Including this burning stage into thermonuclear supernova simulations [46, 76] affects the latest stages of deflagration burning.

\subsection{Example: a highly resolved simulation}

Several simulations based on the implementation described above, both in two and three spatial dimensions, have been presented [77, 45, 38]. In the 2D simulations, numerical convergence in the global quantities was demonstrated. For the implementation on a co-expanding computational grid, a similar result was found [71]. The numerical convergence naturally arises from the interplay of the resolved flame front representation with the turbulent subgrid-scale model. Ideally, a lack of resolution of large-scale features in the flame front representation should be compensated by an increased turbulent flame propagation velocity determined from the subgrid-scale approach. Of course, a certain threshold of resolution will need to be exceeded in order to reach this regime in the numerical implementation. A consistent and reliable sub-grid scale modeling was the goal of a recent highly resolved simulation [48]. This model shall be described here in order to illustrate the typical flame evolution in the deflagration scenarios of thermonuclear supernovae. A full-star [47] multi-spot ignition [25] model was set up on a computational grid comprizing $1024 \times 1024$ cells. In combination with the nested moving grid approach [25] this facilitated an extremely fine-structured initial flame geometry (see the upper left panel of Figure 1).

Starting from this initial flame configuration, the evolution of the flame front in the explosion process is illustrated by snapshots of the $G=0$ isosurface at $t=0.6 \mathrm{~s}$ and $t=3.0 \mathrm{~s}$ in Figure 1 (upper right and lower left panels). The development of the flame shape from ignition to $t=0.6 \mathrm{~s}$ is characterized by the formation of the well-known "mushroom-like" structures resulting from buoyancy. During the flame evolution, inner structures of smaller scales catch up with the outer "mushrooms" and the initially separated structures merge forming a connected configuration (see snapshot at $t=0.6 \mathrm{~s}$ of Fig. 1). The continued development of substructure and the merger of features create a deflagration structure with a complex pattern. Burning and flotation drive the flame towards the surface of the WD. The fuel density drops as the flame moves outwards due to radial stratification and the overall expansion of the WD caused by the energy deposit from nuclear burning. Once the fuel density falls below the threshold for the production of intermediatemass elements, nuclear burning ceases. This occurs first at the leading features of the flame and subsequently in more central flame regions. Finally, no burning takes place anymore. At this point the outer ash features have reached the surface layers of the ejecta (lower left panel of Fig. 1). The following seconds in the evolution are characterized by hydrodynamical relaxation towards homologous expansion. This stage is reached to good approximation at about $10 \mathrm{~s}$ after ignition [71]. 


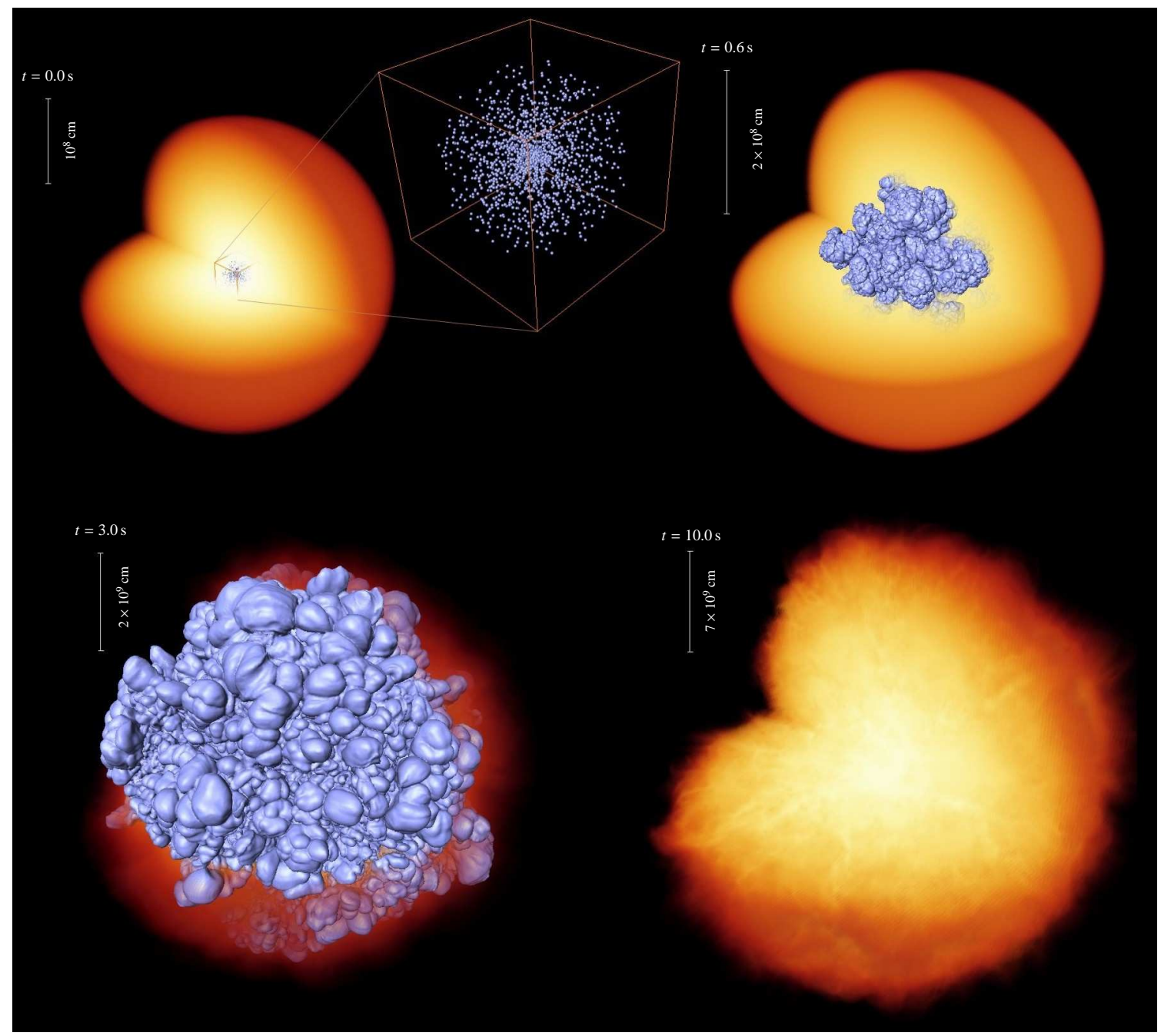

Figure 1: Snapshots from a full-star SN Ia simulation starting from a multi-spot ignition scenario. The logarithm of the density is volume rendered indicating the extend of the WD star and the isosurface corresponds to the thermonuclear flame. The last snapshot corresponds to the end of the simulation and is not on scale with the earlier snapshots (from [48]).

This three-dimensional evolution leads to a remnant of the explosion with characteristic properties. The density structure has patterns from unstable and turbulent flame propagation imprinted on it (see the lower right panel of Fig. 1), and ash regions extend to the outermost layers of the expanding cloud of gas.

\subsection{Comparison with observations}

Due to recent progress in deriving observables from multi-dimensional deflagration simulations, a direct comparison with details of observations of nearby SNe Ia has come into reach. Since the simulations contain no other parameters than the initial conditions, the question arises of whether they meet observational constraints. Such constraints result from the global characteristics derived from observations, observed lightcurves, and spectra taken from nearby SNe Ia. We 


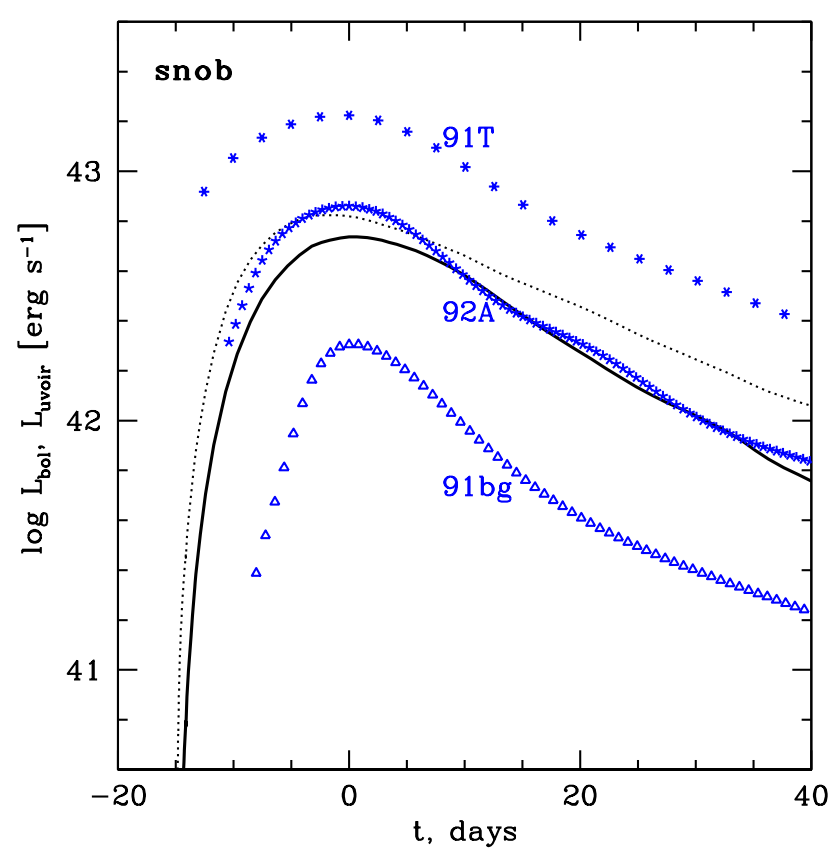

Figure 2: Bolometric lightcurve derived from the highly resolved simulation (black curves; solid is the "UVOIR-bolometric" lightcurve and the complete bolometric lightcurve is dotted). The blue dotted curves correspond to observed bolometric lightcurves [79] (from [48]).

will present a comparison of the highly resolved simulation described above with observational expectations in the following.

The global characteristics derived from SN Ia observations state that a valid explosion model should release $\sim 10^{51} \mathrm{erg}$ of energy and produce about $0.4 \ldots 0.7 \mathrm{M}_{\odot}$ of ${ }^{56} \mathrm{Ni}$ in the nuclear burning $[78,79]$. However, there exists a large diversity in the observations ranging from the class of sub-luminous SNe Ia (like SN $1991 \mathrm{bg}$ with probably $\sim 0.1 \mathrm{M}_{\odot}$ of ${ }^{56} \mathrm{Ni}$ ) to super-luminous events (e.g. SN $1991 \mathrm{~T}$ with a ${ }^{56} \mathrm{Ni}$ mass close to $1 \mathrm{M}_{\odot}$ ). The simulation under consideration here led to an asymptotic kinetic energy of the ejecta of $\sim 8.1 \times 10^{50} \mathrm{erg}$ and produced $\sim 0.6 \mathrm{M}_{\odot}$ of iron group elements. It thus falls into the range of observational expectations, albeit on the weaker side.

Lightcurves - Lightcurves of SNe Ia are sensitive to the energy release, the ${ }^{56} \mathrm{Ni}$ production, as well as to the distribution of elements in the ejecta. In Figure 2 a synthetic bolometric lightcurve derived from the above described simulation is compared with observed lightcurves [53]. It was calculated using the StelLa code $[80,81,82]$. The synthetic bolometric lightcurve falls into the range of observed lightcurves of SNe Ia (indicated in Figure 2 by the extreme examples of the super-luminous SN $1991 \mathrm{~T}$ and the sub-luminous SN 1991bg). With respect to brightness and shape it compares reasonably well with SN 1994D, a standard normal SN Ia.

Spectra - A much harder test for the models is posed by the comparison of synthetic and observed spectra since these depend on details in the composition of the ejected material. A powerful diagnostic tool to compare SN Ia models with spectral observations is provided by the abundance tomography of SN 2002bo [83]. It makes use of spectra taken from this supernova with an ex- 


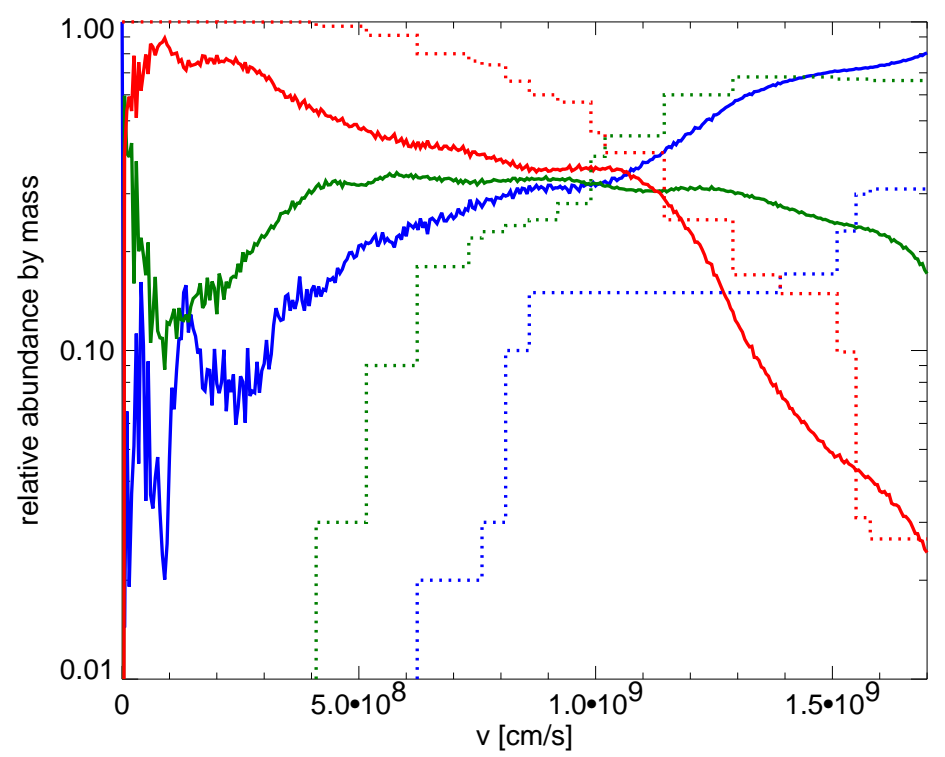

Figure 3: Spherically averaged composition resulting from the hydrodynamical explosion simulation (solid lines) compared to the findings of the abundance tomography of SN 2002bo (dotted lines). Iron group element abundances are shown in red, intermediate-mass elements in green, and unburned material in blue (from [48]).

traordinary good time coverage. Fitting this sequence of data with synthetic spectra unveils the composition of the ejecta in velocity space slice by slice (see Figure 3), since the photosphere moves gradually inwards with the expansion of the remnant.

This abundance tomography of the ejecta can be compared with results of 3D models, when averaged over the angles (Figure 3). Qualitatively, the mixed composition of the ejecta found here is reproduced by deflagration SN Ia models in a natural way since these predict a distribution of burnt material within the rising bubbles. A problem was, however, that older models predicted large unburnt mass fractions in the central parts of the ejecta in disagreement with the observational results $[84,83]$. The high-resolved simulation cures this problem by clearly reproducing the irongroup dominance in the low-velocity ejecta [48]. A good agreement between the simulation and the abundance tomography of SN 2002bo is therefore recovered in the inner parts of the ejecta. Above radii of $\sim 10,000 \mathrm{~km} \mathrm{~s}^{-1}$, however, the simulation predicts the chemical composition to be dominated by unburnt material. This is in contradiction to observations which find the material still to be dominated by nuclear ashes, mainly intermediate-mass elements. It should however be noted that these outer regions of the ejecta contain only little mass $\left(\sim 0.25 \mathrm{M}_{\odot}\right)$ due to the low densities encountered here. Thus, the observed chemical composition is reproduced by the model for the larger part of the ejected material.

\subsection{Summary of the deflagration model}

The pure deflagration model has proven very successful in many respects. It provides the up to now only fully self-consistent thermonuclear supernova model. Apart from the initial conditions (flame ignition, WD structure and composition), which are expected to vary in nature, it describes the actual explosion process without tunable parameters. Perhaps the most striking success is that 
it indeed leads to an explosion of the WD releasing energies that fall into the range of observational expectations. The structure of the ejecta seems to be partially consistent with compositions of SN Ia ejecta derived from spectral observations. At least the inner part of the ejecta looks similar to what is expected for the dimmer examples of normal SNe Ia.

Despite these successes, there are shortcomings of the pure deflagration model. Varying the initial conditions in a range that seems physically plausible, it could not reproduce the full range of observed SN Ia diversity. In particular, the normal to bright examples of the observational SN Ia sample seem to be out of reach for the deflagration model. For these to be reproduced, ${ }^{56} \mathrm{Ni}$ masses of the order of $0.7 \mathrm{M}_{\odot}$ need to be synthesized in the explosion. This could not be achieved thus far in the deflagration model. The second concern is with regard to the composition of the ejecta. The large-scale Rayleigh-Taylor instabilities lead to clumpy inhomogeneities in the structure of the explosion ejecta and to a strong mixing of different groups of reaction products in the angular averaged profile. While the results may still be consistent with observations in the central parts of the ejecta, the chemical composition of the outer parts seems to be at odds with the observation, where an intermediate-mass element dominance of the ejecta is detected out to large radii. It seems that the burning provided by the deflagration flame is insufficient, in particular in late phases of the explosion. Such an incomplete burning would explain the shortcomings in reproducing the outermost parts of the ejecta and the fact that the deflagration model fails to explain the brighter SN Ia events. Although the kinetic energy of the explosion ejecta falls into the range of observational expectations, it reaches only the lower end of what is expected for normal SNe Ia. Two interpretations are possible here:

1. The deflagration model accounts for a certain peculiar sub-class of SNe Ia [85], namely rather dim events which show indications for unburnt material in their ejecta.

2. The deflagration model is incomplete but provides an important building block for an extended explosion model. This would explain why it is rather successful in reproducing the dimmer SN Ia events. For these, the deflagration phase may dominate the explosion process.

In the following section, we elaborate on the second of these possibilities.

\section{The delayed detonation model}

A promising extension of the pure deflagration model is the delayed detonation model [35]. By assuming a transition of the flame propagation mode from subsonic deflagration to supersonic detonation in an advanced stage of the explosion process, it provides a way to accelerate and enhance burning in the late phases. According to the discussion above, this may be a way to achieve a better agreement with SN Ia observations. Not only would it lead to a stronger explosion and to an enrichment of the outer layers of the ejecta with nuclear ashes, but it would also weaken the clumpy inhomogeneities in the ejecta by supersonically burning down the funnels in between the uprising Rayleigh-Taylor plumes.

\subsection{Deflagration-to-detonation transitions in SNe Ia}

The delayed detonation model, however, is still afflicted with severe uncertainties. Certainly the greatest hindrance for its success is that the physical mechanism providing a deflagration-to- 


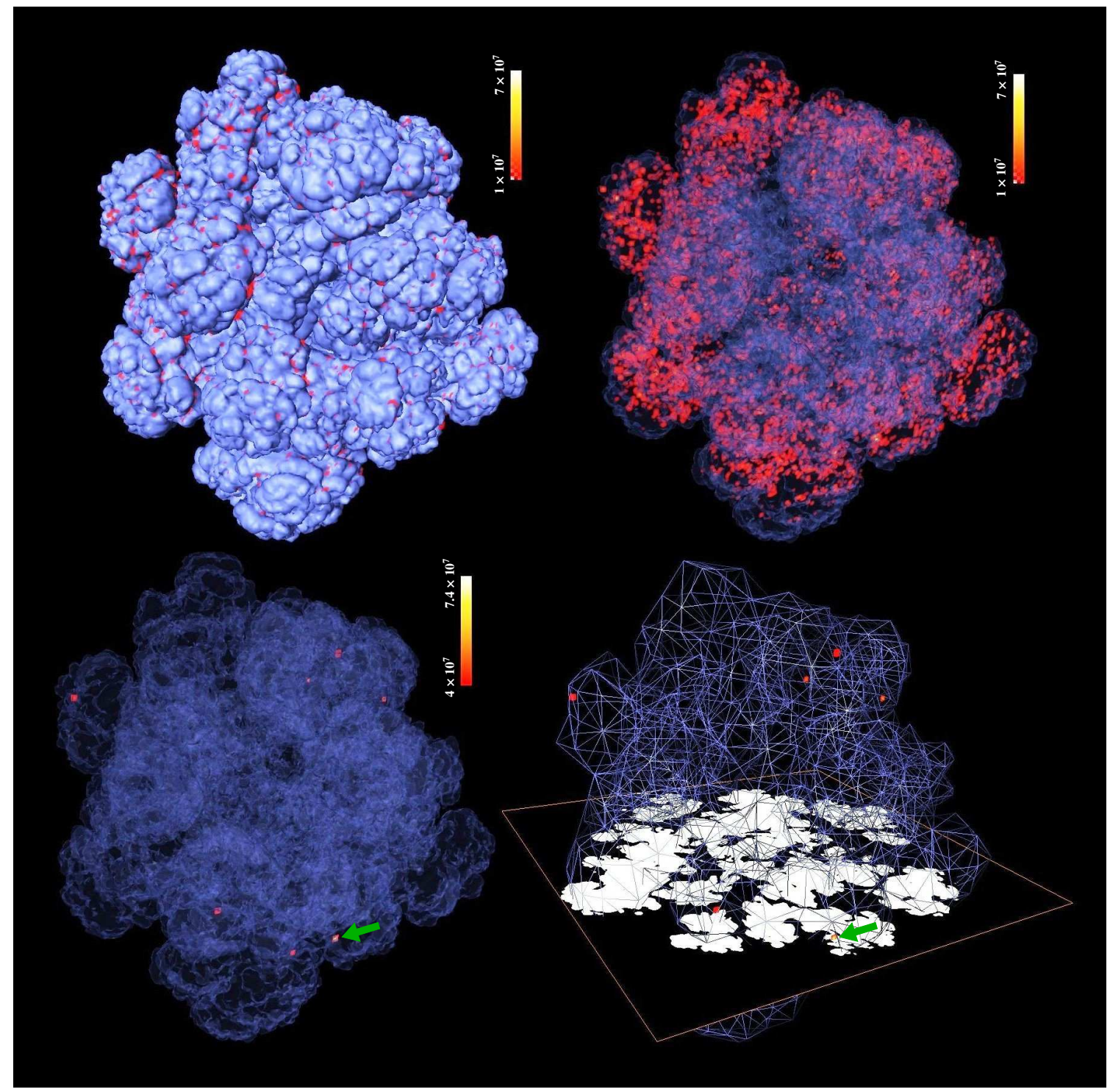

Figure 4: Turbulent velocity fluctuations $v^{\prime}$ at patches of the flame which have entered the distributed burning regime. The blue opaque, transparent, or wire-mesh surfaces correspond to the flame front. Volumes of high turbulent velocity fluctuations are rendered in red/orange. In the lower panels, the green arrow indicates the location of the maximum value of $v^{\prime}$ found in the simulation. For better visibility, white areas correspond to ash regions and fuel regions are shown in black in a plane intersecting with the maximum $v^{\prime}$-value in the lower right panel (from [86]).

detonation transition (DDT henceforth) in thermonuclear supernovae remains unclear [87]. Such transitions are observed in terrestrial combustion, but there they occur in the vicinity of obstacles or at the walls of the combustion vessel. Such boundaries do not exist in the astrophysical situation. Therefore, it seems reasonable to assume that a DDT occurs (if happening at all) in connection with the only significant change in the structure of the deflagration flame that occurs when it enters the distributed burning regime [88]. Remarkably, this takes place at fuel densities of the order $10^{7} \mathrm{~g} \mathrm{~cm}^{-3}$, coinciding with the "transition density" that led to best results in parameterized one- 


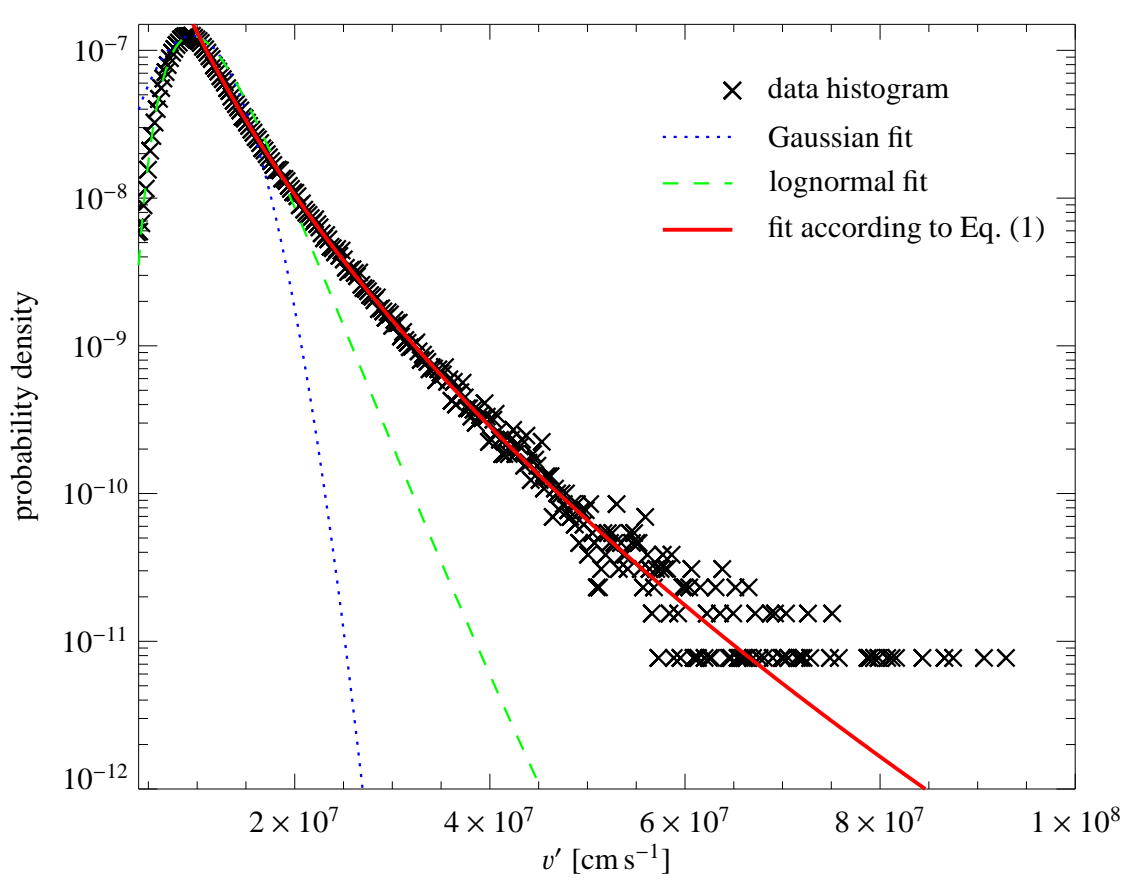

Figure 5: Fits to the histogram of the turbulent velocity fluctuations $v^{\prime}$ (from [86]).

dimensional delayed detonation simulations.

DDTs occurring in the distributed burning regime seem to be possible [89], but only if a very high turbulence intensity is retained in these late explosion processes. Turbulent velocity fluctuations of the order of $1000 \mathrm{~km} \mathrm{~s}^{-1}$ seem to be necessary to trigger a DDT. While earlier twodimensional simulations of the deflagration phase in thermonuclear supernovae failed to reach these values, a recent analysis of three-dimensional simulations [86] found that such high turbulent intensities are likely to be realized in late burning stages. An example is given in Figure 4 illustrating the situation in the highly resolved deflagration simulation described in Sect. 4. Patches of the flame front are color-coded which have entered the distributed burning regime and still feature high turbulent velocities. A histogram of the velocity fluctuations corresponding to the situation of Figure 4 is shown in Figure 5. It features an extended high-velocity tail. An acceptable fit to this histogram is obtained with a probability density function following an exponential of a geometric Ansatz:

$$
P\left(v^{\prime}\right)=\exp \left[a_{0}\left(v^{\prime}\right)^{a_{1}}+a_{2}\right]
$$

For values of the fitting parameters $a_{0}, a_{1}$, and $a_{2}$ see [86]. From such an analysis it is evident that the necessary high turbulent velocity fluctuations are likely to be achieved at non-negligible patches of the flame front and a flame-driven DDT thus cannot be excluded. However, the microscopic mechanism of this transition remains largely unclear. A high value of the mean turbulent velocity is necessary, but certainly not sufficient [90]. Further studies of the microscopic properties of burning in the distributed regime are needed in order to settle the question of a DDT in thermonuclear supernovae. 


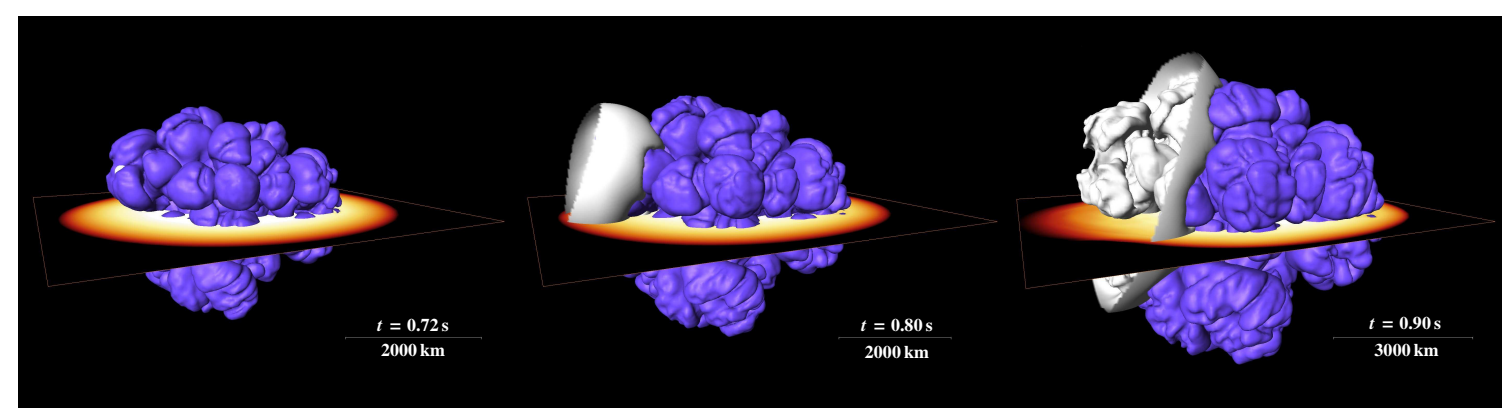

Figure 6: Initiation and propagation of the detonation wave (white isosurface) in a delayed detonation model [53]. The deflagration flame is shown as blue isosurface and the extent of the star is indicated by the central plane mapping the logarithm of the density (from [53]).

\subsection{Delayed detonations in full-star simulations}

Despite the uncertainties of DDTs, an approach to address the question of delayed detonations in thermonuclear supernovae is to assume such a transition and to artificially trigger the detonation wave in large simulations. The question to be addressed by this approach is whether the outcome of such models is consistent with observations. Prescribing the DDT to occur at an arbitrary instant and a location near the center led to promising results [51]. However, such an approach suffers from the arbitrariness of the assumed DDT and has little predictive power.

A way to assess the potential of the delayed detonation model is to fix the DDT to some physically motivated guess [53]. According to the discussion above, one possibility is to assume the DDT to occur at the location where the flame first enters the distributed burning regime [52, 53]. Although this is a very simplifying assumption, it fixes the unknown DDT parameter and allows to compare the results of the simulations with observational expectations. This was done both in two-dimensional [52] and three-dimensional [53] simulations. Particular care was taken to prevent the detonation from crossing ashes left behind by the previous deflagration stage. This requirement follows from a recent analysis of the propagation of detonations in WD matter [91]. It can easily be implemented by following the detonation wave with a level-set approach [52, 28, 92].

A typical evolution of a three-dimensional full-star simulation is shown in Figure 6. After ignition, the deflagration phase proceeds in a way similar to the pure deflagration model. The burning bubbles grow by flame propagation and rise buoyantly towards the surface of the WD. Due to instabilities and partial merger of the bubbles, a complex connected structure develops. The parametrized DDT criterion is met first at $0.724 \mathrm{~s}$ after ignition at the outer edge of the flame front. Here, the detonation is triggered by initiating the corresponding level set, as shown in the left panel of Fig. 6. The fact that the outer edge of the deflagration flame is selected by the imposed DDT criterion is not surprising. Turbulence is generated preferentially at large buoyant bubbles and at the same time the density is lowest at the outermost parts of the flame making the flame broadest here. This favors the transition to the distributed burning regime and thus the parametrized initiation of the detonation wave.

The center and right panels of Fig. 6 show the propagation of the detonation wave. Since it cannot cross ash regions, it wraps around the corrugated deflagration structure burning towards the star's center. In this way, it takes about $0.2 \mathrm{~s}$ before it arrives at the center of the WD. Meanwhile, 
the star keeps expanding and the deflagration continues in regions not yet reached by the detonation wave. Consequently, the density of the fuel ahead of the detonation drops quickly after passing the WD's center. This way, burning stalls shortly before the detonation reaches the far side of the deflagration structure. Nonetheless, this still implies burning of most of the WD, since these deflagration features have already reached the low density edge of the star.

Such simulations emphasize that the propagation of the detonation wave has to compete with the expansion of the WD caused by the previous deflagration phase. The outcome of this competition with respect to the completeness of the burning, the ${ }^{56} \mathrm{Ni}$ production (an thus the brightness of the resulting event), as well as the energy release depends strongly on the efficiency of burning in the deflagration phase. This can be modified by varying the flame ignition geometry [53] and leads to models that are capable of covering the global characteristics of normal to bright observed SNe Ia [53]. Weak deflagrations lead to less expansion of the WD before the detonation is triggered and the detonation thus encounters a large amount of unburnt material at relatively high densities. Therefore it significantly contributes to the overall burning leading to very bright and energetic events dominated by the detonation phase. In strong deflagrations, in contrast, the high-density burning takes place almost exclusively in the deflagration phase. The detonation thus contributes little to the mass of radioactive nickel resulting in a rather dim and moderately energetic explosion which bears the imprint of the deflagration phase. Interestingly, however, the detonation at lower densities leads to a layer of intermediate mass elements surrounding the iron-group rich ejecta. Changing the flame ignition (for instance by assuming a multi-spot ignition scenario $[25,26]$ ) it is possible to smoothly shift the characteristics of the result from a deflagration-type explosion to a detonation-dominated event.

\section{Conclusions}

As a conclusion, a speculation on the overall picture of thermonuclear supernovae as a model of SNe Ia can be provided based on [93]. Spectra of a set of well-observed SNe Ia allowed to derive the composition of the ejecta of the events. The result is shown in Figure 7 and indicates that the mass of stable iron group elements is roughly constant for all events $\left(\sim 0.2 \mathrm{M}_{\odot}\right)$. The mass of radioactive ${ }^{56} \mathrm{Ni}$ determines (as expected) the brightness, and the total mass of iron group elements shows a linear correlation with brighness. Interestingly, in all supernovae roughly the same total mass of burnt material is found, i.e. the dimmer events compensate less iron group elements in the ejecta by a larger mass of intermediate-mass elements.

In the theoretical picture outlined above, a possible interpretation is that the dimmer examples of the normal events (corresponding to the central part of the diagram in Figure 7 around $\Delta m_{15}(B) \sim 1.5 \mathrm{mag}$ ) could be accounted for by pure deflagrations. However, because of the difficulty to synthesize large amounts of intermediate mass elements in the outer layers of the ejecta in deflagration models, an alternative interpretation in the context of delayed detonations seems more realistic. As discussed in Sect. 5.2, changing the flame ignition configuration these models facilitates a smooth shift from the characteristics of the model being dominated by the deflagration phase to a detonation-dominated event. In this picture, the SNe Ia in the central part of the diagram in Figure 7 could be explained by the weaker and dimmer models with emphasis on the deflagration phase while the detonation-dominated models seem promising for reproducing the bright and ener- 


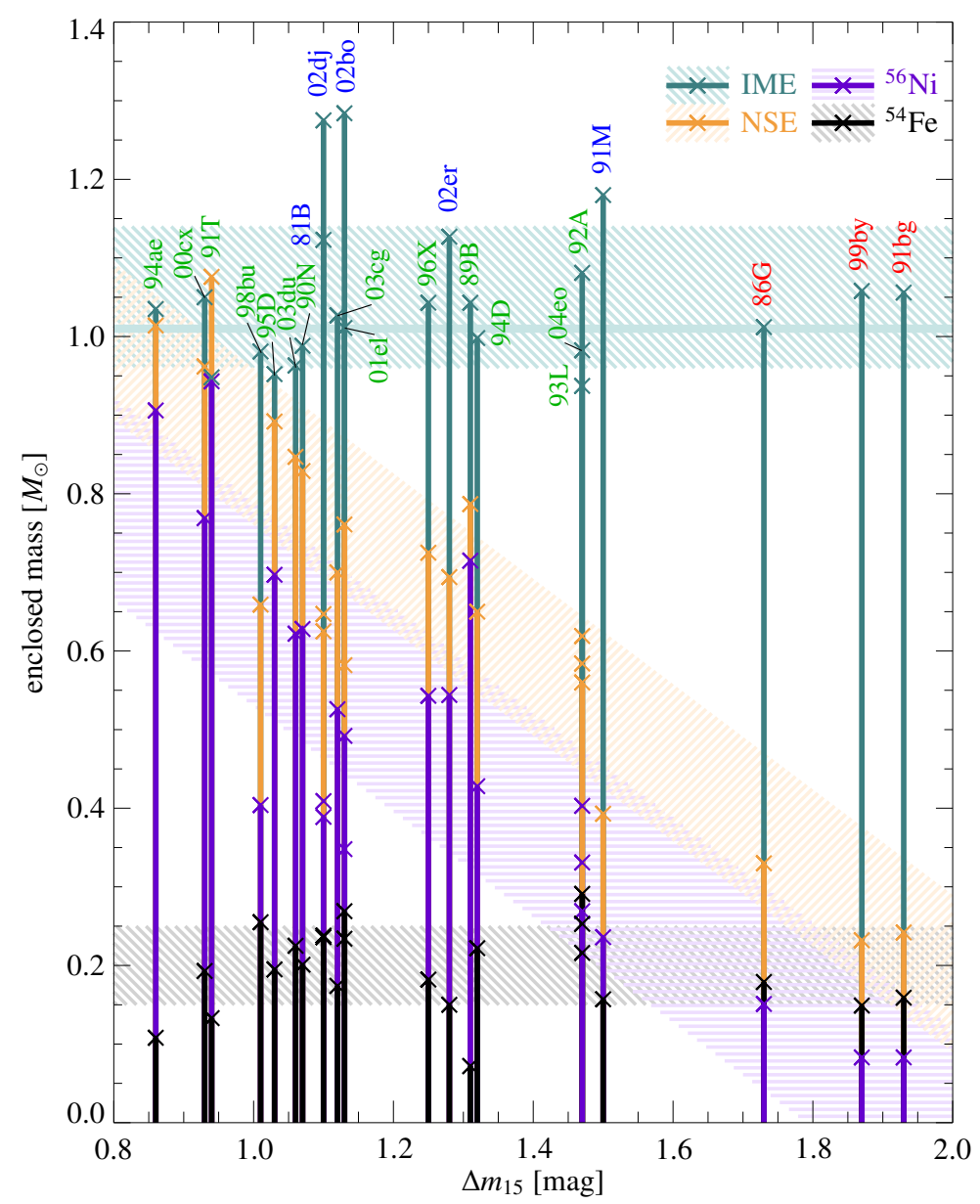

Figure 7: Distribution of the principal isotopic groups in SNe Ia. The enclosed mass (linked to velocity via the W7 explosion model) of different burning products is shown versus decline-rate parameter $\Delta m_{15}(B)$ (a proxy for SN luminosity). The mass of stable ${ }^{54} \mathrm{Fe}+{ }^{58} \mathrm{Ni}$ for each $\mathrm{SN}$ is indicated in black; that of ${ }^{56} \mathrm{Ni}$ is shown in purple, and the sum of these (total NSE mass) is indicated in orange colors. Turquoise crosses show the sum of NSE and IME mass, indicating the total mass burned. The IME mass is given in turquoise color (from [93]).

getic events (on the left-hand side of the plot in Figure 7). In this hypothetic picture, however, the peculiar sub-luminous SNe Ia located to the right in the plot remain unexplained. Further modeling efforts and a detailed derivation of synthetic observables from the results of numerical simulations will provide a way of testing this conjectured scenario. Alternative thermonuclear supernova models, such as gravitationally confined detonations and pulsational reverse detonations, are examined on the basis of comparison with observations as well. While the former scenario seems to generically lead to very bright events only, a potential problem of the latter model is the large amount of iron-group elements in the outer layer of the ejecta [94]. Clearly, further exploration seems necessary to settle the question whether these models can reproduce normal SNe Ia or lead to peculiar events. 


\section{Acknowledgments}

I would like to thank the organizers of the conference for the kind invitation. I enjoyed the inspiring atmosphere at the conference.

\section{References}

[1] P. François, F. Matteucci, R. Cayrel, M. Spite, F. Spite et al. The evolution of the Milky Way from its earliest phases: Constraints on stellar nucleosynthesis. AEFA, 421 (2004) 613. [arXiv: astro-ph/0401499].

[2] D. Branch and G. A. Tammann. Type Ia supernovae as standard candles. ARAEA, 30 (1992) 359.

[3] A. G. Riess, A. V. Filippenko, P. Challis, A. Clocchiatti, A. Diercks et al. Observational Evidence from Supernovae for an Accelerating Universe and a Cosmological Constant. AJ, 116 (1998) 1009. [arXiv: astro-ph/9805201].

[4] S. Perlmutter, G. Aldering, G. Goldhaber, R. A. Knop, P. Nugent et al. Measurements of Omega and Lambda from 42 High-Redshift Supernovae. ApJ, 517 (1999) 565. [arXiv:astro-ph/9812133].

[5] B. Leibundgut. Cosmological Implications from Observations of Type Ia Supernovae. ARAE $A, 39$ (2001) 67.

[6] P. Astier, J. Guy, N. Regnault, R. Pain, E. Aubourg et al. The Supernova Legacy Survey: measurement of $\Omega_{\mathrm{M}}, \Omega_{\Lambda}$ and $w$ from the first year data set. AEA A, 447 (2006) 31. [arXiv: astro-ph/0510447].

[7] W. M. Wood-Vasey, G. Miknaitis, C. W. Stubbs, S. Jha, A. G. Riess et al. Observational Constraints on the Nature of Dark Energy: First Cosmological Results from the ESSENCE Supernova Survey. ApJ, 666 (2007) 694. [arXiv: astro-ph/0701041].

[8] J. C. Wheeler and R. P. Harkness. Type I supernovae. Reports of Progress in Physics, $\mathbf{5 3}$ (1990) 1467.

[9] A. V. Filippenko. Optical Spectra of Supernovae. ARAEA, 35 (1997) 309.

[10] F. Zwicky. On Collapsed Neutron Stars. ApJ, 88 (1938) 522.

[11] F. Hoyle and W. A. Fowler. Nucleosynthesis in Supernovae. ApJ, 132 (1960) 565.

[12] J. W. Truran, W. D. Arnett and A. G. W. Cameron. Nucleosynthesis in supernova shock waves. Canadian Journal of Physics, 45 (1967) 2315.

[13] S. A. Colgate and C. McKee. Early Supernova Luminosity. ApJ, 157 (1969) 623.

[14] W. Hillebrandt and J. C. Niemeyer. Type Ia Supernova Explosion Models. ARAEAA, 38 (2000) 191. [arXiv: astro-ph/0006305]. 
[15] J. Whelan and I. J. Iben. Binaries and Supernovae of Type I. ApJ, 186 (1973) 1007.

[16] K. Nomoto. Accreting white dwarf models for type I supernovae. I. Presupernova evolution and triggering mechanisms. ApJ, 253 (1982) 798.

[17] I. Iben, Jr. and A. V. Tutukov. Supernovae of type I as end products of the evolution of binaries with components of moderate initial mass ( $M$ not greater than about 9 solar masses). ApJS, 54 (1984) 335.

[18] P. Ruiz-Lapuente, F. Comeron, J. Méndez, R. Canal, S. J. Smartt et al. The binary progenitor of Tycho Brahe's 1572 supernova. Nature, 431 (2004) 1069. [arXiv: astro-ph/0410673].

[19] K. Nomoto and I. Iben, Jr. Carbon ignition in a rapidly accreting degenerate dwarf-A clue to the nature of the merging process in close binaries. ApJ, 297 (1985) 531.

[20] D. Garcia-Senz and S. E. Woosley. Type Ia Supernovae: The Flame Is Born. ApJ, 454 (1995) 895.

[21] S. E. Woosley, S. Wunsch and M. Kuhlen. Carbon Ignition in Type Ia Supernovae: An Analytic Model. ApJ, 607 (2004) 921. [arXiv: astro-ph/0307565].

[22] L. Iapichino, M. Brüggen, W. Hillebrandt and J. C. Niemeyer. The ignition of thermonuclear flames in type Ia supernovae. AEA, 450 (2006) 655. [arXiv: astro-ph/0512300].

[23] M. Kuhlen, S. E. Woosley and G. A. Glatzmaier. Carbon Ignition in Type Ia Supernovae. II. A Three-dimensional Numerical Model. ApJ, 640 (2006) 407. [arXiv: astro-ph/0509367].

[24] P. Höflich and J. Stein. On the Thermonuclear Runaway in Type Ia Supernovae: How to Run Away? ApJ, 568 (2002) 779. [arXiv: astro-ph/0104226].

[25] F. K. Röpke, W. Hillebrandt, J. C. Niemeyer and S. E. Woosley. Multi-spot ignition in type Ia supernova models. AEA, 448 (2006) 1. [arXiv: astro-ph/0510474].

[26] W. Schmidt and J. C. Niemeyer. Thermonuclear supernova simulations with stochastic ignition. AEFA, 446 (2006) 627. [arXiv: astro-ph/0510427].

[27] T. Plewa, A. C. Calder and D. Q. Lamb. Type Ia Supernova Explosion: Gravitationally Confined Detonation. ApJ, 612 (2004) L37. [arXiv: astro-ph/0405163].

[28] F. K. Röpke, S. E. Woosley and W. Hillebrandt. Off-Center Ignition in Type Ia Supernovae. I. Initial Evolution and Implications for Delayed Detonation. ApJ, 660 (2007) 1344. [arXiv: astro-ph/0609088].

[29] S. A. Sim, D. N. Sauer, F. K. Röpke and W. Hillebrandt. Light curves for off-centre ignition models of Type Ia supernovae. MNRAS, 378 (2007) 2. [arXiv : astro-ph/0703764].

[30] W. Hillebrandt, S. A. Sim and F. K. Röpke. Off-center explosions of Chandrasekhar-mass white dwarfs: an explanation of super-bright type Ia supernovae? AEFA, 465 (2007) L17. [arXiv:astro-ph/0702344]. 
[31] W. D. Arnett. A Possible Model of Supernovae: Detonation of ${ }^{12}$ C. ApESSS, 5 (1969) 180.

[32] K. Nomoto, D. Sugimoto and S. Neo. Carbon deflagration supernova, an alternative to carbon detonation. ApEsSS, 39 (1976) L37.

[33] F. X. Timmes and S. E. Woosley. The conductive propagation of nuclear flames. I. Degenerate $\mathrm{C}+\mathrm{O}$ and $\mathrm{O}+\mathrm{Ne}+\mathrm{Mg}$ white dwarfs. ApJ, 396 (1992) 649.

[34] L. N. Ivanova, V. S. Imshennik and V. M. Chechetkin. Pulsation regime of the thermonuclear explosion of a star's dense carbon core. ApESSS, 31 (1974) 497.

[35] A. M. Khokhlov. Delayed detonation model for type Ia supernovae. A\&A, 245 (1991) 114.

[36] S. E. Woosley and T. A. Weaver. Massive stars, supernovae, and nucleosynthesis. In S. A. Bludman, R. Mochkovitch and J. Zinn-Justin, eds., Les Houches Session LIV: Supernovae, pp. 63-154. North-Holland, Amsterdam, 1994.

[37] D. Arnett and E. Livne. The delayed-detonation model of Type Ia supernovae. II. The detonation phase. ApJ, 427 (1994) 330.

[38] M. Reinecke, W. Hillebrandt and J. C. Niemeyer. Three-dimensional simulations of type Ia supernovae. AEAA, 391 (2002) 1167. [arXiv: astro-ph/0206459].

[39] V. N. Gamezo, A. M. Khokhlov, E. S. Oran, A. Y. Chtchelkanova and R. O. Rosenberg. Thermonuclear Supernovae: Simulations of the Deflagration Stage and Their Implications. Science, 299 (2003) 77. [arXiv: astro-ph/0212054].

[40] F. K. Röpke. Multi-dimensional numerical simulations of type Ia supernova explosions. In S. Röser, ed., Reviews in Modern Astronomy, Reviews in Modern Astronomy, vol. 19, pp. 127-156. 2006.

[41] F. K. Röpke and W. Hillebrandt. Three-dimensional Modeling of Type Ia Supernova Explosions. In S. Kubono, W. Aoki, T. Kajino, T. Motobayashi and K. Nomoto, eds., Origin of Matter and Evolution of Galaxies, American Institute of Physics Conference Series, vol. 847, pp. 190-195. 2006.

[42] C. Travaglio, W. Hillebrandt, M. Reinecke and F.-K. Thielemann. Nucleosynthesis in multidimensional SN Ia explosions. AEFA, 425 (2004) 1029. [arXiv : astro-ph/0406281].

[43] F. K. Röpke, M. Gieseler, M. Reinecke, C. Travaglio and W. Hillebrandt. Type Ia supernova diversity in three-dimensional models. AEA A, 453 (2006) 203. [arXiv: astro-ph/0506107].

[44] K. Nomoto, F.-K. Thielemann and K. Yokoi. Accreting white dwarf models of Type I supernovae. III. Carbon deflagration supernovae. ApJ, 286 (1984) 644.

[45] M. Reinecke, W. Hillebrandt and J. C. Niemeyer. Refined numerical models for multidimensional type Ia supernova simulations. AEA, 386 (2002) 936. [arXiv: astro-ph/0111475]. 
[46] F. K. Röpke and W. Hillebrandt. The distributed burning regime in type Ia supernova models. $A \mathcal{F} A, 429$ (2005) L29. [arXiv: astro-ph/0411667].

[47] F. K. Röpke and W. Hillebrandt. Full-star type Ia supernova explosion models. AEFA, 431 (2005) 635. [arXiv: astro-ph/0409286].

[48] F. K. Röpke, W. Hillebrandt, W. Schmidt, J. C. Niemeyer, S. I. Blinnikov et al. A ThreeDimensional Deflagration Model for Type Ia Supernovae Compared with Observations. ApJ, 668 (2007) 1132. [arXiv:0707.1024].

[49] F. K. Röpke, W. Hillebrandt and J. C. Niemeyer. The cellular burning regime in type Ia supernova explosions. I. Flame propagation into quiescent fuel. AEFA, 420 (2004) 411. [arXiv: astro-ph/0312092].

[50] F. K. Röpke, W. Hillebrandt and S. I. Blinnikov. On the Mechanism of Type Ia Supernovae. In ESA Special Publication, ESA Special Publication, vol. 637. 2006.

[51] V. N. Gamezo, A. M. Khokhlov and E. S. Oran. Three-dimensional Delayed-Detonation Model of Type Ia Supernovae. ApJ, 623 (2005) 337. [arXiv: astro-ph/0409598].

[52] I. Golombek and J. C. Niemeyer. A model for multidimensional delayed detonations in SN Ia explosions. AEA A, 438 (2005) 611. [arXiv: astro-ph/0503617].

[53] F. K. Röpke and J. C. Niemeyer. Delayed detonations in full-star models of type Ia supernova explosions. AEA A, 464 (2007) 683.

[54] E. Bravo and D. Garcia-Senz. A Three-Dimensional Picture of the Delayed-Detonation Model of Type Ia Supernovae. ArXiv e-prints, 712 (2007). [arXiv: 0712.0510].

[55] T. Plewa. Detonating Failed Deflagration Model of Thermonuclear Supernovae. I. Explosion Dynamics. ApJ, 657 (2007) 942. [arXiv: astro-ph/0611776].

[56] D. M. Townsley, A. C. Calder, S. M. Asida, I. R. Seitenzahl, F. Peng et al. Flame Evolution During Type Ia Supernovae and the Deflagration Phase in the Gravitationally Confined Detonation Scenario. ApJ, 668 (2007) 1118. [arXiv: 0706. 1094].

[57] F. K. Röpke and S. E. Woosley. Surface detonation in type Ia supernova explosions? Journal of Physics Conference Series, 46 (2006) 413. [arXiv : astro-ph/0609691].

[58] E. Bravo and D. García-Senz. Beyond the Bubble Catastrophe of Type Ia Supernovae: Pulsating Reverse Detonation Models. ApJ, 642 (2006) L157. [arXiv: astro-ph/0604025].

[59] F. K. Röpke, J. C. Niemeyer and W. Hillebrandt. On the Small-Scale Stability of Thermonuclear Flames in Type Ia Supernovae. ApJ, 588 (2003) 952. [arXiv: astro-ph/0211202].

[60] F. K. Röpke, W. Hillebrandt and J. C. Niemeyer. The cellular burning regime in type Ia supernova explosions. II. Flame propagation into vortical fuel. AEFA, 421 (2004) 783. [arXiv:astro-ph/0312203]. 
[61] M. Zingale, S. E. Woosley, C. A. Rendleman, M. S. Day and J. B. Bell. Three-dimensional Numerical Simulations of Rayleigh-Taylor Unstable Flames in Type Ia Supernovae. ApJ, 632 (2005) 1021. [arXiv: astro-ph/0501655].

[62] W. Schmidt, W. Hillebrandt and J. C. Niemeyer. Level set simulations of turbulent thermonuclear deflagration in degenerate carbon and oxygen. Combust. Theory Modelling, 9 (2005) 693. [arXiv: astro-ph/0508076].

[63] N. Peters. Turbulent Combustion. Cambridge University Press, Cambridge, 2000.

[64] A. Khokhlov. Flame Modeling in Supernovae. ApJ, 419 (1993) L77.

[65] A. C. Calder, T. Plewa, N. Vladimirova, D. Q. Lamb and J. W. Truran. Type Ia Supernovae: An Asymmetric Deflagration Model (2004). ArXiv:astro-ph/0405126.

[66] M. Reinecke, W. Hillebrandt, J. C. Niemeyer, R. Klein and A. Gröbl. A new model for deflagration fronts in reactive fluids. AEA, 347 (1999) 724. [arXiv: astro-ph/9812119].

[67] S. Osher and J. A. Sethian. Fronts Propagating with Curvature-Dependent Speed: Algorithms Based on Hamilton-Jacobi Formulations. Journal of Computational Physics, 79 (1988) 12.

[68] V. Smiljanovski, V. Moser and R. Klein. A capturing-tracking hybrid scheme for deflagration discontinuities. Combustion Theory Modelling, 1 (1997) 183.

[69] W. Hillebrandt, M. Reinecke, W. Schmidt, F. K. Röpke, C. Travaglio et al. Simulations of turbulent thermonuclear burning in Type Ia supernovae. In G. Warnecke, ed., Analysis and Numerics for Conservation Laws, pp. 363-384. Springer, Berlin Heidelberg New York, 2005. [arXiv: astro-ph/0405209].

[70] F. K. Röpke and W. Hillebrandt. The case against the progenitor's carbon-to-oxygen ratio as a source of peak luminosity variations in type Ia supernovae. AEA, 420 (2004) L1. [arXiv: astro-ph/0403509].

[71] F. K. Röpke. Following multi-dimensional type Ia supernova explosion models to homologous expansion. AEAA, 432 (2005) 969. [arXiv: astro-ph/0408296].

[72] W. Schmidt, J. C. Niemeyer, W. Hillebrandt and F. K. Röpke. A localised subgrid scale model for fluid dynamical simulations in astrophysics. II. Application to type Ia supernovae. AEAA, 450 (2006) 283. [arXiv: astro-ph/0601500].

[73] J. C. Niemeyer and A. R. Kerstein. Burning regimes of nuclear flames in SN Ia explosions. New Astronomy, 2 (1997) 239.

[74] G. Damköhler. Der Einfluß der Turbulenz auf die Flammengeschwindigkeit in Gasgemischen. Z. f. Elektroch., 46 (1940) 601.

[75] J. C. Niemeyer and W. Hillebrandt. Microscopic Instabilities of Nuclear Flames in Type Ia Supernovae. ApJ, 452 (1995) 779. 
[76] W. Schmidt. On the applicability of the level set method beyond the flamelet regime in thermonuclear supernova simulations. AEA, 465 (2007) 263. [arXiv: astro-ph/0701416].

[77] M. Reinecke, W. Hillebrandt and J. C. Niemeyer. Thermonuclear explosions of Chandrasekhar-mass $C+O$ white $\quad d$ warfs. $\quad A \mathcal{E} A, \quad 347 \quad$ (1999) 739. [arXiv: astro-ph/9812120].

[78] G. Contardo, B. Leibundgut and W. D. Vacca. Epochs of maximum light and bolometric light curves of type Ia supernovae. AEA, 359 (2000) 876. [arXiv: astro-ph/0005507].

[79] M. Stritzinger, B. Leibundgut, S. Walch and G. Contardo. Constraints on the progenitor systems of type Ia supernovae. AEA, 450 (2006) 241. [arXiv: astro-ph/0506415].

[80] S. I. Blinnikov, R. Eastman, O. S. Bartunov, V. A. Popolitov and S. E. Woosley. A Comparative Modeling of Supernova 1993J. ApJ, 496 (1998) 454. [arXiv: astro-ph/9711055].

[81] S. I. Blinnikov and E. I. Sorokina. UV light curves of thermonuclear supernovae. AEAA, 356 (2000) L30. [arXiv: astro-ph/0003247].

[82] S. I. Blinnikov, F. K. Röpke, E. I. Sorokina, M. Gieseler, M. Reinecke et al. Theoretical light curves for deflagration models of type Ia supernova. AEA, 453 (2006) 229. [arXiv: astro-ph/0603036].

[83] M. Stehle, P. A. Mazzali, S. Benetti and W. Hillebrandt. Abundance stratification in Type Ia supernovae - I. The case of SN 2002bo. MNRAS, 360 (2005) 1231. [arXiv: astro-ph/0409342].

[84] C. Kozma, C. Fransson, W. Hillebrandt, C. Travaglio, J. Sollerman et al. Three-dimensional modeling of type Ia supernovae - The power of late time spectra. AEA, 437 (2005) 983. [arXiv: astro-ph/0504317].

[85] M. M. Phillips, W. Li, J. A. Frieman, S. I. Blinnikov, D. DePoy et al. The Peculiar SN 2005hk: Do Some Type Ia Supernovae Explode as Deflagrations? PASP, 119 (2007) 360. [arXiv: astro-ph/0611295].

[86] F. K. Röpke. Flame-driven Deflagration-to-Detonation Transitions in Type Ia Supernovae? ApJ, 668 (2007) 1103. [arXiv: 0709. 4095].

[87] J. C. Niemeyer. Can Deflagration-Detonation Transitions Occur in Type Ia Supernovae? ApJ, 523 (1999) L57. [arXiv: astro-ph/9906142].

[88] J. C. Niemeyer and S. E. Woosley. The Thermonuclear Explosion of Chandrasekhar Mass White Dwarfs. ApJ, 475 (1997) 740. [arXiv : astro-ph/9607032].

[89] A. M. Lisewski, W. Hillebrandt and S. E. Woosley. Constraints on the Delayed Transition to Detonation in Type Ia Supernovae. ApJ, 538 (2000) 831. [arXiv: astro-ph/9910056].

[90] S. E. Woosley. Type Ia Supernovae: Burning and Detonation in the Distributed Regime. ApJ, 668 (2007) 1109. [arXiv: 0709 . 4237]. 
[91] A. Maier and J. C. Niemeyer. $C+O$ detonations in thermonuclear supernovae: interaction with previously burned material. AEA 451 (2006) 207. [arXiv: astro-ph/0605293].

[92] M. Fink, W. Hillebrandt and F. K. Röpke. Double-detonation supernovae of subChandrasekhar mass white dwarfs. AESA, 476 (2007) 1133. [arXiv: 0710. 5486].

[93] P. A. Mazzali, F. K. Röpke, S. Benetti and W. Hillebrandt. A Common Explosion Mechanism for Type Ia Supernovae. Science, 315 (2007) 825. [arXiv: astro-ph/0702351].

[94] E. Baron, D. J. Jeffery, D. Branch, E. Bravo, D. García-Senz et al. Detailed Spectral Modeling of a Three-dimensional Pulsating Reverse Detonation Model: Too Much Nickel. ApJ, 672 (2008) 1038. [arXiv:0709.4177]. 